\title{
Spectra of High-Frequency Waves in Solar Coronal Plasma
}

\author{
V. G. Ledenev ${ }^{1}$ and V. V. Tirsky ${ }^{2}$ \\ ${ }^{1}$ Institute of Solar-Terrestrial Physics SD RAS, P.O.Box 4026, 664033, Irkutsk, Russia, email: \\ leden@iszf.irk.ru \\ ${ }^{2}$ Institute of Laser Physics SD RAS, Irkutsk Branch, Irkutsk, Russia
}

To obtain the wave characteristics of hot magnetized plasma, requires investigating a complete dispersion equation describing the oscillations of magnetized plasma, of the form

$$
a+\left(\omega^{2} / k^{2} c^{2}\right) b+\left(\omega^{4} / k^{4} c^{4}\right) c_{0}=0
$$

Here,

$$
\begin{gathered}
a=\left(k_{\perp}^{2} / k^{2}\right) \varepsilon_{x x}+\left(k_{z}^{2} / k^{2}\right) \varepsilon_{z z}+2\left(k_{\perp} k_{z} / k^{2}\right) \varepsilon_{x z} \\
b=-\varepsilon_{x x} \varepsilon_{z z}+\varepsilon_{x z}^{2}-\left(k_{z}^{2} / k^{2}\right)\left(\varepsilon_{y y} \varepsilon_{z z}+\varepsilon_{y z}^{2}\right)-\left(k_{\perp}^{2} / k^{2}\right)\left(\varepsilon_{x x} \varepsilon_{y y}+\varepsilon_{x y}^{2}\right)+ \\
2\left(k_{\perp} k_{z} / k^{2}\right)\left(\varepsilon_{x y} \varepsilon_{y z}-\varepsilon_{x z} \varepsilon_{z y}\right) \\
c_{0}=\varepsilon_{z z}\left(\varepsilon_{x x} \varepsilon_{y y}+\varepsilon_{x y}^{2}\right)+\varepsilon_{x x} \varepsilon_{y z}^{2}-\varepsilon_{y y} \varepsilon_{x z}^{2}+2 \varepsilon_{y z} \varepsilon_{x z} \varepsilon_{x y}
\end{gathered}
$$

$\omega$ is the complex frequency of the wave, $\mathrm{k}$ is the wave number, $\mathrm{c}$ is the velocity of light in a vacuum, $k_{z}$ is the longitudinal (with respect to the magnetic field) wave vector component, $k_{\perp}$ is the transverse wave vector component, and $\varepsilon_{i j}$ stands for the components of the dielectric tensor.

In this paper we investigate numerically the high-frequency oscillations $\left(\omega>\omega_{H e}\right)$ of hot magnetized plasma with refractive indices $n \leqslant 10$. Figures a-d present the results of calculations of the dependence of the refractive index $n$ on the frequency of weakly damped modes (1 - x-mode, 2 - o-mode, 3 - z-mode) for the angles between the wave vector and the magnetic field $\theta=0 ; 0.2 ; 1$, and 1.5 for the parameters $\omega_{p e} / \omega_{H e}=$ 10 , and $v_{T e}^{2} / c^{2}=2 \cdot 10^{-4}$. These values are consistent with parameters of the solar corona. The summation with respect to cyclotron harmonics was made up to the fifteenth harmonic. The dispersion equation for transverse waves propagating along magnetic field for $\theta=0$ and $\omega^{2} \gg \omega_{H e}^{2}$ is $n^{2}=1-\omega_{p e}^{2} \omega_{H e} / \omega^{3} \pm \omega_{p e}^{2} \omega_{H e} / \omega^{3}$. The upper sign corresponds to $\mathrm{x}$-mode and the lower one - to o-mode. Setting $\mathrm{n}=0$, we get the cut-off frequency for o-mode $\omega_{c o}=\left(2 \omega_{p e}^{2} \omega_{H e}\right)^{1 / 3}$. This value agrees well with our numerical result (Figure a). For angles $\theta>1$ the dispersion curves intersect and the waves transform from one type to another one (Figure d). Given the plasma parameters, the frequency of the intersection point is close to the cut-off frequency for x-mode $\omega_{c}$. In its turn from our calculation we have $\omega_{c} \approx 5 \omega_{p e}$ or $\omega_{c} \approx \omega_{p e}^{2} / 2 \omega_{H e}$.

The results allow us to explain more naturally such peculiarities in solar radio emission as the splitting of type II bursts, mutual position harmonic components of type III bursts and others.

\section{Splitting of type II bursts}

We suggest that type II bursts are generated in the moving magnetic traps (arches) in the solar corona. As we know, electron beams with anisotropic distribution function are formed in such magnetic configurations. As a consequence, the beam-anisotropic instability develops and plasma waves propagating across magnetic field are excited. 


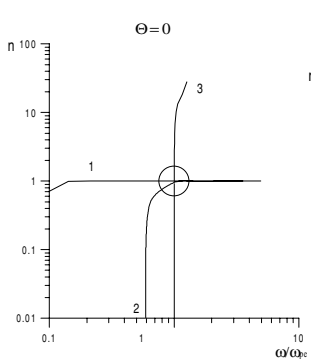

a

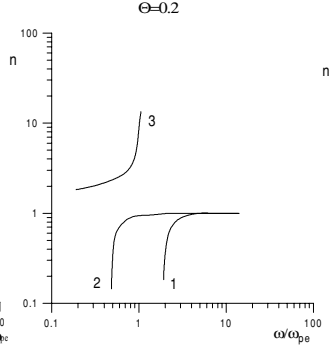

b

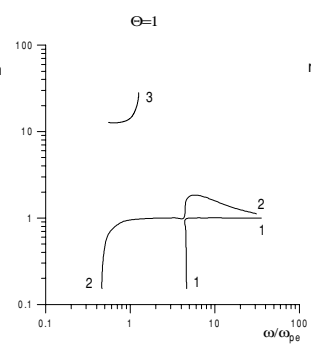

c

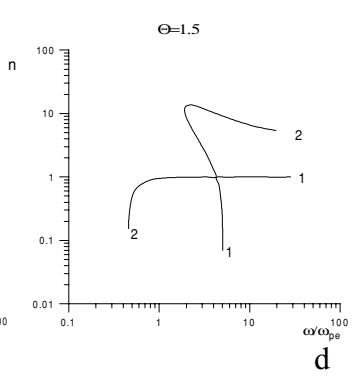

Figure 1.

As is seen from our calculations (Figure 1d), the wave frequencies are about two times higher than the local plasma frequency for $n=10$. The emission can escape directly along branches 1 and 2 (fundamental component) as well as a result of plasma waves coalescence process (harmonic component). As one can see from Figure 1 (d) one electron beam can excite plasma waves at two different frequencies. These frequencies correspond to branches with positive and negative dispersion. This effect is observed as the splitting of type II burst components. If electron stream has interrupted character then so named "herring-bone" structure is generated.

\section{Mutual position of fundamental and harmonic components sources in type III bursts}

If the source of type III burst moves in picture plane its emission at a fixed frequency escapes from two levels in solar corona. To the first of them (lower) corresponds the emission with frequency close to local plasma frequency. The emission is generated as a result of plasma wave coalescence (harmonic component). The emission, which escapes from second (higher) level, has the frequency close to doubled local plasma frequency (Figure 1d). This emission is generated by anisotropic electron beam and propagates mainly across the magnetic field. If the emission from first level is generated at the harmonic of plasma frequency then the emission from second level corresponds to fundamental component because the local plasma frequency at this level about two times lower. The observations at fixed frequency but at different moments of time therewith will show approximate coincidence of fundamental and harmonic component sources positions. On the other hand the simultaneous observations at frequencies differ in two times will show that the harmonic component source is situated on deeper level in the corona than the fundamental component source.

This work was supported by RFBR grants 03-02-16229, 477.2003.2. 